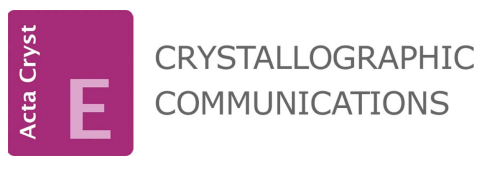

ISSN 2056-9890

Received 19 October 2018

Accepted 28 January 2019

Edited by W. T. A. Harrison, University of Aberdeen, Scotland

Keywords: crystal structure; thiazolidin-4-one; tin complex; $\mathrm{C}-\mathrm{H}$...Cl-metal hydrogen bond.

CCDC reference: 1894217

Supporting information: this article has supporting information at journals.iucr.org/e

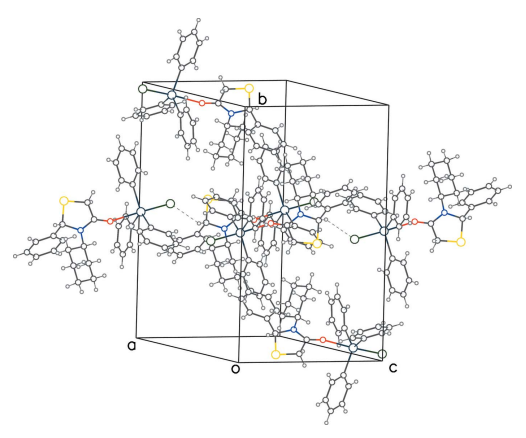

\section{Crystal structure of a 1:1 adduct of triphenyltin chloride with 3-cyclohexhyl-2-phenyl-1,3-thia- zolidin-4-one}

\author{
Hemant P. Yennawar, ${ }^{a}$ John Tierney ${ }^{\mathrm{b}}$ and Kevin C. Cannon ${ }^{\mathrm{c} *}$ \\ ${ }^{a}$ The Pennsylvania State University, Department of Biochemistry and Molecular Biology, University Park, PA 16802, USA, \\ ${ }^{\mathbf{b}}$ Pennsylvania State University, Brandywine Campus, Department of Chemistry, Brandywine, PA 19063, USA, and ${ }^{\mathbf{C}}$ The \\ Pennsylvania State University, Department of Chemistry, Abington College, Abington, PA 19001, USA. *Correspondence \\ e-mail: kcc10@psu.edu
}

In the centrosymmetric (racemic) title compound, chlorido(3-cyclohexhyl-2phenyl-1,3-thiazolidin-4-one- $\kappa O)$ triphenyltin(IV), $\left[\mathrm{Sn}\left(\mathrm{C}_{6} \mathrm{H}_{5}\right)_{3} \mathrm{Cl}\left(\mathrm{C}_{15} \mathrm{H}_{19} \mathrm{NOS}\right)\right]$, the tin(IV) atom exhibits a trigonal-bipyramidal coordination geometry with the three phenyl groups in equatorial positions and the chloride anion and ligand oxygen atom present at axial sites $\left[\mathrm{O}-\mathrm{Sn}-\mathrm{Cl}=175.07(14)^{\circ}\right]$. The thiazolidinone ring of the ligand adopts an envelope conformation with the $\mathrm{S}$ atom as the flap. The dihedral angles between the heterocycle ring plane (all atoms) are $44.3(9)^{\circ}$ with respect to the pendant $C$-phenyl plane and $34.3(11)^{\circ}$ to the $N$-cyclohexyl ring (all atoms). The $C$-phenyl and $N$-cyclohexyl ring are close to orthogonal to each other, with a dihedral angle of $81.1(4)^{\circ}$ between them. In the crystal, molecules are linked by weak $\mathrm{C}-\mathrm{H} \cdots \mathrm{Cl}$ hydrogen bonds to generate [001] chains.

\section{Chemical context}

Substituted 1,3-thiazolidin-4-ones themselves as well as ligands attached to various metals exhibit a wide range of biological activity (Jain et al., 2012; Kozlowski et al. 2002). The ligand of the title compound, $(N)$-3-xyclohexyl-2-phenyl-1,3thiazolidine-4-one, is easily prepared from $N$-cyclcohexylidene aniline and thioglycolic acid utilizing a method originally proposed by Surrey (1947). The crystal structure of $(N)-3-$ cyclohexyl-2-phenyl-1,3-thiazolidine-4-one has previously been reported (Cannon et al. 2013), as have a number of other 2,3-disubstituted-thiazolidin-4-one structures (Yennawar et al., 2017; Vigorita et al., 1979). Furthermore, the X-ray crystal structure of 2,3-diphenyl-1,3-thiazolidin-4-one as a 1:1 adduct with triphenyltin chloride has been described (Smith et al. 1995), and along with related complexes has biological activity against Cerotysistis Ulmi, the fungus that causes Dutch Elm Disease (Beraldo \& de Lima, 2008).

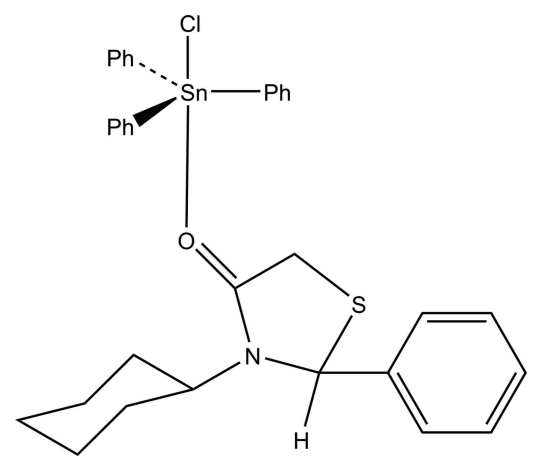




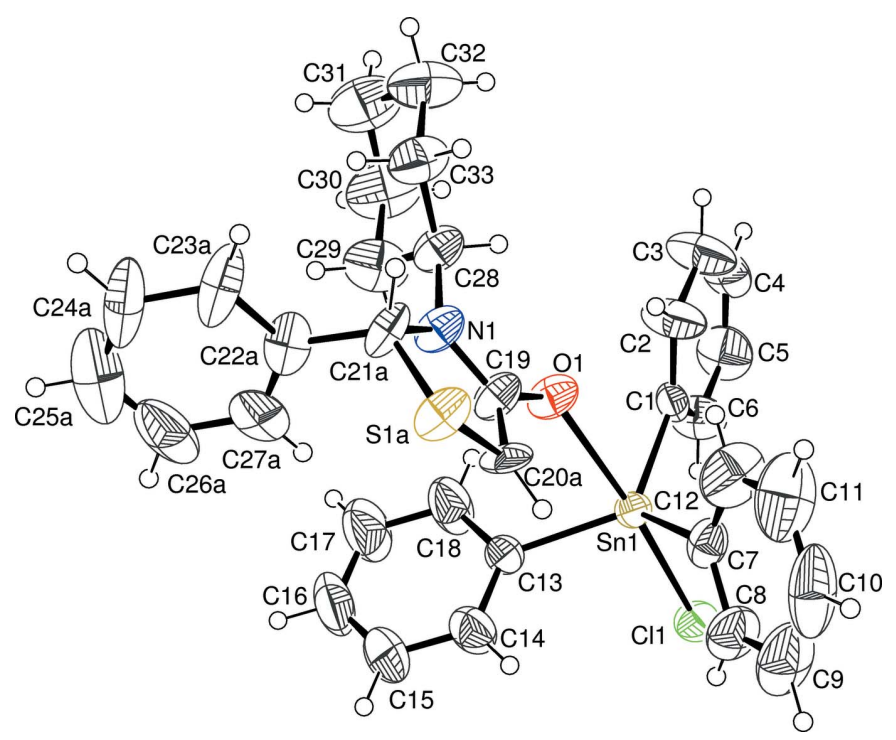

Figure 1

The molecular structure of the title compound with displacement ellipsoids drawn at the $40 \%$ probability level. Only one disorder component of the thiazolidinone ring and its attached $\mathrm{C} 22$ phenyl ring are shown.

Herein, we report the synthesis and crystal structure of the 1:1 adduct of triphenyltin chloride with $(N)$-3-cyclohexhyl-2phenyl-1,3-thiazolidin-4-one.
Table 1

Selected bond lengths $(\AA)$.

\begin{tabular}{llll}
\hline Sn1-C1 & $2.141(4)$ & Sn1-Cl1 & $2.4439(19)$ \\
Sn1-C7 & $2.130(4)$ & Sn1-O1 & $2.488(4)$ \\
Sn1-C13 & $2.119(4)$ & & \\
\hline
\end{tabular}

\section{Structural commentary}

The title compound (Fig. 1) shows a five-coordinate geometry around the tin atom (Table 1) with three phenyl groups placed equatorially, and a chloride ligand and an O-bonded thiazolidinone ligand at the axial sites. The $\mathrm{Cl}-\mathrm{Sn}-\mathrm{O}$ (ligand) principal axis is almost $5^{\circ}$ off its ideal linear geometry with a bond angle of $175.07(14)^{\circ}$. The $(N)$-3-cyclohexhyl-2-phenyl1,3-thiazolidin-4-one ligand contains a chiral center at the 2-carbon atom (C21): in the arbitrarily chosen asymmetric unit, this atom has an $R$ configuration, but crystal symmetry generates a racemic mixture.

The most closely related structure previously reported is that of 2,3-diphenyl-1,3-thiazolidin-4-one as a 1:1 adduct with triphenyltin chloride (Smith et al., 1995). Since this molecule had a less bulky phenyl group at N3 (N1 in our numbering scheme) than the more bulky cyclohexyl group, the principal angle is almost exactly linear at $179.2^{\circ}$. Previously, using Mössbauer effect spectroscopy, the 2,3-diphenyl-1,3-thia-

Figure 2

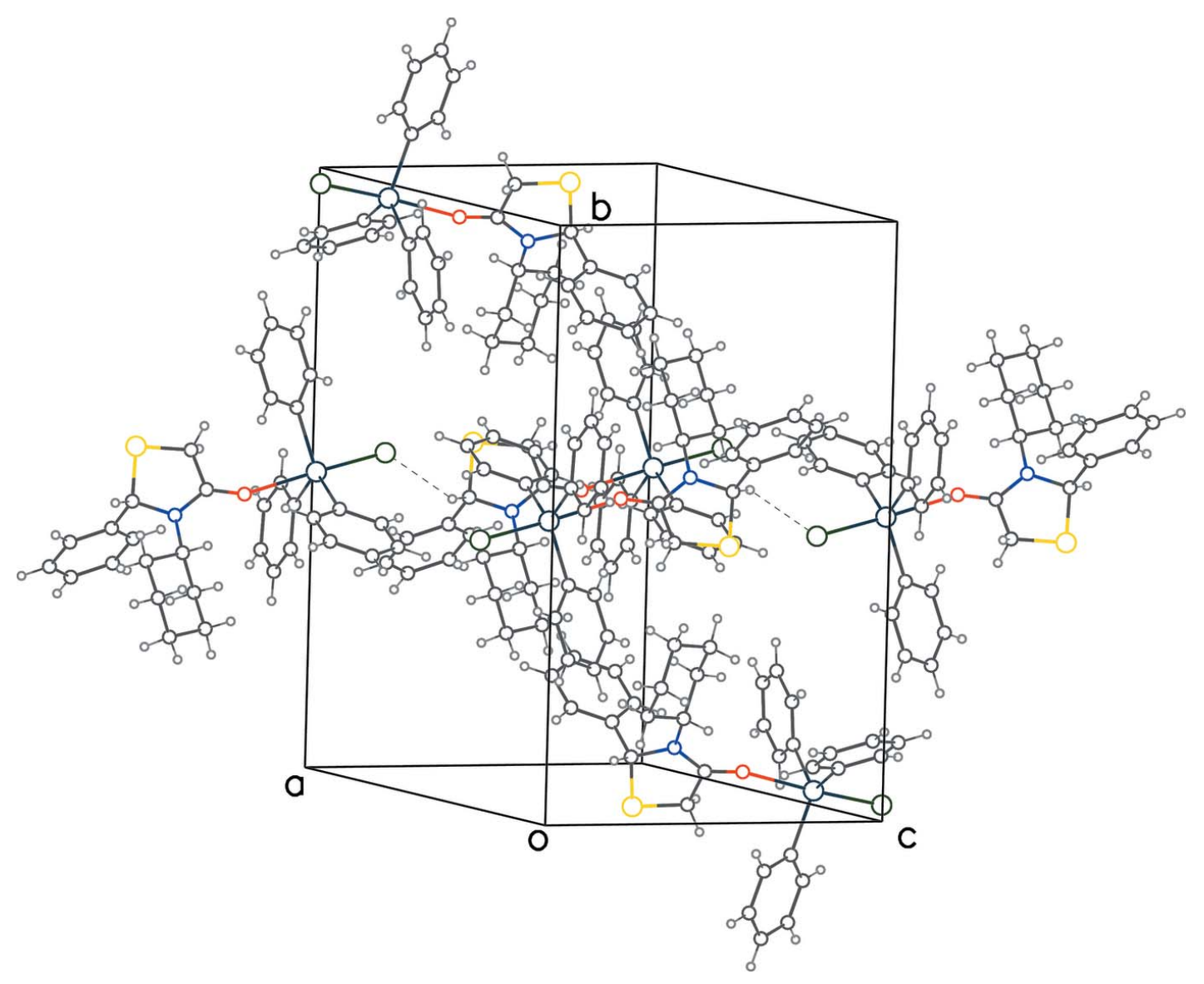

Packing diagram for the title compound with $\mathrm{C}-\mathrm{H} \cdots \mathrm{Cl}$ interactions indicated by dashed lines. 
zolidin-4-one as a 1:1 adduct with triphenyltin chloride gave an $r$ value (the ratio of quadrupole splitting to isomer shift) of 2.41 , indicative of the tin with a coordination number greater than four. Although Mössbauer spectroscopy was not used in our study, we see the same coordination properties with the title molecule in the $\mathrm{X}$-ray structure. The $\mathrm{Sn}-\mathrm{O}$ bond length was found to be $2.500 \AA$ for the tin-diphenylthiazolidinone adduct, using Mössbauer techniques as well as the X-ray data, whereas, the X-ray data for the title compound yields an $\mathrm{Sn}-$ O bond length of 2.488 (4) $\AA$. These values are almost the same and show no difference in having the presence of phenyl and a cyclohexyl group at $\mathrm{C} 2$ and N3 (C21 and N1 in our numbering scheme) versus a phenyl group at each location.

\section{Supramolecular features}

The surface of the title compound is primarily hydrophobic due to four aromatic and one aliphatic ring resulting in intermolecular van der Waals interactions (Fig. 2) between the various aromatic rings. A sole weak hydrogen bond between the chiral carbon atom (C21) with a chloride ion of the neighboring molecule related by translation symmetry in the $c$-axis direction $[\mathrm{H} \cdots \mathrm{Cl}=2.76 \AA, \mathrm{C} \cdots \mathrm{Cl}=3.569$ (9) $\AA, \mathrm{C}-$ $\left.\mathrm{H} \cdots \mathrm{Cl}=140^{\circ}\right]$ helps to consolidate the packing.

\section{Database survey}

There is only one closely related structure previously reported and that is 2,3-diphenyl-1,3-thiazolidin-4-one as a 1:1 adduct with triphenyltin chloride (Smith et al., 1995).

\section{Synthesis and crystallization}

The synthesis of $(N)$-3-cyclohexyl-2-phenyl-1,3-thiazolidine-4one has been previously reported (Cannon et al., 2013).

The 1:1 adduct with triphenyltin chloride was prepared by dissolving $0.0023 \mathrm{~mol}$ of $\mathrm{N}$-3-cyclohexhyl-2-phenyl-1,3-thiazolidin-4-one in $15 \mathrm{ml}$ of acetone and adding this solution dropwise to a $15 \mathrm{~mL}$ solution of triphenyltin chloride $(0.0023 \mathrm{~mol})$ in a $50 \mathrm{ml}$ round-bottom flask while stirring at room temperature for $3 \mathrm{~h}$. Stirring was then stopped and the solution was allowed to stand for an additional $10 \mathrm{~h}$. A precipitate was apparent, which was filtered and the filtrate was reduced under vacuum on a rotary evaporator, dried under vacuum to give an oily residue, which formed crystals when heated in ligroin. Recrystallization from ligroin solution yielded $0.0022 \mathrm{~mol}$ ( $97 \%$ yield) of the title $1: 1$ complex in the form of colorless blocks: m.p. 372-375 K (no literature reports).

Triphenyltinchloride-3-cyclohexyl-2-phenyl-1,3-thiazolidin4-one: Yield (97\%); m.p. $372-375 \mathrm{~K}, \mathrm{~cm}^{-1} 1658.6(\mathrm{C}=\mathrm{O}) ;{ }^{1} \mathrm{H}$ $\operatorname{NMR}\left(\mathrm{CDCl}_{3}\right): 7.78-7.27(20 \mathrm{H}, m$, aromatics $), 5.66(1 \mathrm{H}, d, J=$ $1.9 \mathrm{~Hz}, \mathrm{C} 2), 3.89(1 \mathrm{H}, d d, J=1.9 \mathrm{~Hz}$ and $J=15.6 \mathrm{~Hz}, \mathrm{C} 5), 3.85-$ $3.78(1 \mathrm{H}, m, \mathrm{NCH}), 3.58(1 \mathrm{H}, d, J=15.6 \mathrm{~Hz}, \mathrm{C} 5), 1.79-0.91$ (10H, $m$, cyclohexyls); ${ }^{13} \mathrm{C}$ NMR: 171.77 (C4), 142.98, 137.78, $136.34(t, 25.3 \mathrm{~Hz}), 130.62,129.32(t, J=32.2 \mathrm{~Hz}), 129.07$,
Table 2

Experimental details.

\begin{tabular}{|c|c|}
\hline \multicolumn{2}{|l|}{ Crystal data } \\
\hline Chemical formula & {$\left[\mathrm{Sn}\left(\mathrm{C}_{6} \mathrm{H}_{5}\right)_{3} \mathrm{Cl}\left(\mathrm{C}_{15} \mathrm{H}_{19} \mathrm{NOS}\right)\right]$} \\
\hline$M_{\mathrm{r}}$ & 646.81 \\
\hline Crystal system, space group & Monoclinic, $P 2_{1} / c$ \\
\hline Temperature (K) & 218 \\
\hline$a, b, c(\AA)$ & $15.360(5), 18.879(6), 10.992(3)$ \\
\hline$\beta\left(^{\circ}\right)$ & $102.524(5)$ \\
\hline$V\left(\mathrm{~A}^{3}\right)$ & $3111.8(17)$ \\
\hline$Z$ & 4 \\
\hline Radiation type & Мo $K \alpha$ \\
\hline$\mu\left(\mathrm{mm}^{-1}\right)$ & 1.00 \\
\hline Crystal size $(\mathrm{mm})$ & $0.15 \times 0.11 \times 0.10$ \\
\hline \multicolumn{2}{|l|}{ Data collection } \\
\hline Diffractometer & Bruker CCD area detector \\
\hline Absorption correction & $\begin{array}{l}\text { Multi-scan (SADABS, Bruker, } \\
\text { 2001) }\end{array}$ \\
\hline$T_{\min }, T_{\max }$ & $0.865,0.907$ \\
\hline $\begin{array}{l}\text { No. of measured, independent and } \\
\text { observed }[I>2 \sigma(I)] \text { reflections }\end{array}$ & 24296, 7791, 5009 \\
\hline$R_{\text {int }}$ & 0.072 \\
\hline$(\sin \theta / \lambda)_{\max }\left(\AA^{-1}\right)$ & 0.673 \\
\hline \multicolumn{2}{|l|}{ Refinement } \\
\hline$R\left[F^{2}>2 \sigma\left(F^{2}\right)\right], w R\left(F^{2}\right), S$ & $0.083,0.221,1.04$ \\
\hline No. of reflections & 7791 \\
\hline No. of parameters & 365 \\
\hline No. of restraints & 133 \\
\hline $\mathrm{H}$-atom treatment & $\mathrm{H}$-atom parameters constrained \\
\hline$\Delta \rho_{\max }, \Delta \rho_{\min }\left(\mathrm{e} \AA^{-3}\right)$ & $2.50,-1.17$ \\
\hline
\end{tabular}

Computer programs: SMART and SAINT (Bruker, 2001), SHELXS97 and SHELXL97 (Sheldrick, 2008) and OLEX2 (Dolomanov et al., 2009).

128.88, 128.52, 126.38, 62.83 (C2), 56.30, 33.23 (C5), 31.03, 30.12, 26.10, 25.42. $\mathrm{C}_{33} \mathrm{H}_{34} \mathrm{OClSnNS}$.

\section{Refinement}

In spite of our search for a better crystal we had to work with one that was not optimal, as is evident from the high value of $R_{\text {int }}=0.0721$. Upon refinement we observed positional disorder in almost a fourth of the structure (nine out of thirtyeight non-H atoms). As a result, some refinement parameters such as the ADP max/min ratio (8.2) for one of the atoms are slightly above optimal values but the atomic connectivity is clearly established. Crystal data, data collection and structure refinement details are summarized in Table 2. The $\mathrm{H}$ atoms were placed geometrically and allowed to ride on their parent $\mathrm{C}$ atoms during refinement, with $\mathrm{C}-\mathrm{H}$ distances of $0.93 \AA$ (aromatic) and $0.97 \AA$ (methylene), with $U_{\text {iso }}(\mathrm{H})=1.2 U_{\text {eq }}$ (aromatic or methylene C) or $1.5 U_{\text {eq }}$ (methyl C).

\section{Acknowledgements}

We thank Temple University, Department of Chemistry, for the use of their Bruker $500 \mathrm{MHz}$ NMR spectrometer.

\section{Funding information}

Funding for this research was provided by: NSF funding (CHEM-0131112) for the X-ray diffractometer . 


\section{References}

Beraldo, H. \& de Lima, G. M. (2008). Tin Chemistry: Fundamentals, Frontiers and Applications, edited by A. Davies, M. Gielen, K. H. Pannell \& E. R. T. Tiekink, p 448. Chichester: John Wiley \& Sons. Bruker (2001). SMART, SAINT and SADABS. Bruker AXS Inc., Madison, Wisconsin, USA.

Cannon, K., Mascavage, L., Kistler, K., Tierney, J., Yennawar, H. \& Lagalante, A. (2013). Int. J. Chem. 5, 46-56.

Dolomanov, O. V., Bourhis, L. J., Gildea, R. J., Howard, J. A. K. \& Puschmann, H. (2009). J. Appl. Cryst. 42, 339-341.
Jain, A. K., Vaidya, A., Ravichandran, V., Kashaw, S. K. \& Agrawal, R. K. (2012). Bioorg. Med. Chem. 20, 3378-3395.

Kozlowski, C. A., Ulewicz, M., Walkowiak, W., Girek, T. J. \& Jablonska, J. (2002). Miner. Eng. 15, 677-682.

Sheldrick, G. M. (2008). Acta Cryst. A64, 112-122.

Smith, F. E., Hynes, R. C., Tierney, J., Zhang, Z. \& Eng, G. (1995). Can. J. Chem. 73, 95-99.

Surrey, A. R. (1947). J. Am. Chem. Soc. 69, 2911-2.

Vigorita, M. G., Chimirri, A., Grasso, S. \& Fenech, G. (1979). J. Heterocycl. Chem. 16, 1257-1261.

Yennawar, H. P., Tierney, J. \& Silverberg, L. J. (2017). IUCrData, 2, x171662. 


\section{supporting information}

Acta Cryst. (2019). E75, 338-341 [https://doi.org/10.1107/S2056989019001592]

\section{Crystal structure of a 1:1 adduct of triphenyltin chloride with 3-cyclohexhyl-2-} phenyl-1,3-thiazolidin-4-one

\section{Hemant P. Yennawar, John Tierney and Kevin C. Cannon}

Computing details

Data collection: SMART (Bruker, 2001); cell refinement: SAINT (Bruker, 2001); data reduction: SAINT (Bruker, 2001); program(s) used to solve structure: SHELXS97 (Sheldrick, 2008); program(s) used to refine structure: SHELXL97 (Sheldrick, 2008); molecular graphics: OLEX2 (Dolomanov et al., 2009); software used to prepare material for publication: OLEX2 (Dolomanov et al., 2009).

Chlorido(3-cyclohexhyl-2-phenyl-1,3-thiazolidin-4-one- $\kappa O$ )triphenyltin(IV)

Crystal data

$\left[\mathrm{Sn}\left(\mathrm{C}_{6} \mathrm{H}_{5}\right)_{3} \mathrm{Cl}\left(\mathrm{C}_{15} \mathrm{H}_{19} \mathrm{NOS}\right)\right]$

$M_{r}=646.81$

Monoclinic, $P 2{ }_{1} / c$

$a=15.360(5) \AA$

$b=18.879(6) \AA$

$c=10.992(3) \AA$

$\beta=102.524(5)^{\circ}$

$V=3111.8(17) \AA^{3}$

$Z=4$

\section{Data collection}

Bruker CCD area detector diffractometer

Radiation source: fine-focus sealed tube Parallel-graphite monochromator phi and $\omega$ scans

Absorption correction: multi-scan

(SADABS, Bruker, 2001)

$T_{\min }=0.865, T_{\max }=0.907$

Refinement

Refinement on $F^{2}$

Least-squares matrix: full

$R\left[F^{2}>2 \sigma\left(F^{2}\right)\right]=0.083$

$w R\left(F^{2}\right)=0.221$

$S=1.04$

7791 reflections

365 parameters

133 restraints

Primary atom site location: structure-invariant direct methods
$F(000)=1320$

$D_{\mathrm{x}}=1.381 \mathrm{Mg} \mathrm{m}^{-3}$

Mo $K \alpha$ radiation, $\lambda=0.71073 \AA$

Cell parameters from 4375 reflections

$\theta=2.3-26.4^{\circ}$

$\mu=1.00 \mathrm{~mm}^{-1}$

$T=218 \mathrm{~K}$

Block, colorless

$0.15 \times 0.11 \times 0.10 \mathrm{~mm}$

24296 measured reflections

7791 independent reflections

5009 reflections with $I>2 \sigma(I)$

$R_{\text {int }}=0.072$

$\theta_{\max }=28.6^{\circ}, \theta_{\min }=1.7^{\circ}$

$h=-16 \rightarrow 20$

$k=-25 \rightarrow 25$

$l=-14 \rightarrow 14$

Secondary atom site location: difference Fourier map

Hydrogen site location: inferred from neighbouring sites

$\mathrm{H}$-atom parameters constrained

$w=1 /\left[\sigma^{2}\left(F_{\mathrm{o}}^{2}\right)+(0.0926 P)^{2}+6.5369 P\right]$

where $P=\left(F_{\mathrm{o}}^{2}+2 F_{\mathrm{c}}^{2}\right) / 3$

$(\Delta / \sigma)_{\max }<0.001$

$\Delta \rho_{\max }=2.50 \mathrm{e} \AA^{-3}$

$\Delta \rho_{\min }=-1.16 \mathrm{e}^{-3}$ 


\section{Special details}

Geometry. All esds (except the esd in the dihedral angle between two 1.s. planes) are estimated using the full covariance matrix. The cell esds are taken into account individually in the estimation of esds in distances, angles and torsion angles; correlations between esds in cell parameters are only used when they are defined by crystal symmetry. An approximate (isotropic) treatment of cell esds is used for estimating esds involving l.s. planes.

Refinement. Refinement of $\mathrm{F}^{2}$ against ALL reflections. The weighted R-factor $\mathrm{wR}$ and goodness of fit $\mathrm{S}$ are based on $\mathrm{F}^{2}$, conventional $R$-factors $R$ are based on $F$, with $F$ set to zero for negative $F^{2}$. The threshold expression of $F^{2}>2 \operatorname{sigma}\left(\mathrm{F}^{2}\right)$ is used only for calculating R-factors(gt) etc. and is not relevant to the choice of reflections for refinement. R-factors based on $\mathrm{F}^{2}$ are statistically about twice as large as those based on F, and R- factors based on ALL data will be even larger.

Fractional atomic coordinates and isotropic or equivalent isotropic displacement parameters $\left(\hat{A}^{2}\right)$

\begin{tabular}{|c|c|c|c|c|c|}
\hline & $x$ & $y$ & $z$ & $U_{\text {iso }} * / U_{\text {eq }}$ & Occ. $(<1)$ \\
\hline $\mathrm{C} 1$ & $0.5942(3)$ & 0.0290 & $0.2921(4)$ & $0.0467(14)$ & \\
\hline $\mathrm{C} 2$ & $0.5376(4)$ & $0.0374(4)$ & $0.1758(4)$ & $0.092(3)$ & \\
\hline $\mathrm{H} 2$ & 0.5556 & 0.0224 & 0.1045 & $0.111^{*}$ & \\
\hline $\mathrm{C} 3$ & $0.4542(4)$ & $0.0681(4)$ & $0.1662(5)$ & $0.116(4)$ & \\
\hline $\mathrm{H} 3$ & 0.4163 & 0.0738 & 0.0884 & $0.139^{*}$ & \\
\hline $\mathrm{C} 4$ & $0.4274(3)$ & $0.0905(4)$ & $0.2728(7)$ & 0.089 & \\
\hline $\mathrm{H} 4$ & 0.3715 & 0.1111 & 0.2664 & $0.106^{*}$ & \\
\hline $\mathrm{C} 5$ & $0.4839(4)$ & $0.0821(4)$ & $0.3891(5)$ & $0.097(3)$ & \\
\hline H5 & 0.4660 & 0.0971 & 0.4604 & $0.116^{*}$ & \\
\hline C6 & $0.5674(4)$ & 0.0513 & $0.3987(4)$ & $0.077(2)$ & \\
\hline H6 & 0.6052 & 0.0457 & 0.4765 & $0.092 *$ & \\
\hline $\mathrm{C} 7$ & $0.7266(4)$ & $-0.1279(2)$ & $0.2527(5)$ & $0.0585(17)$ & \\
\hline $\mathrm{C} 12$ & $0.6759(5)$ & $-0.1492(3)$ & $0.1380(5)$ & 0.108 (4) & \\
\hline H12 & 0.6395 & -0.1167 & 0.0872 & $0.130^{*}$ & \\
\hline C11 & $0.6795(5)$ & -0.2190 & $0.0993(6)$ & $0.132(4)$ & \\
\hline H11 & 0.6455 & -0.2333 & 0.0225 & $0.158^{*}$ & \\
\hline $\mathrm{C} 10$ & $0.7338(6)$ & $-0.2676(2)$ & $0.1753(8)$ & $0.126(4)$ & \\
\hline H10 & 0.7362 & -0.3143 & 0.1494 & $0.151^{*}$ & \\
\hline C9 & $0.7845(5)$ & $-0.2463(3)$ & $0.2900(8)$ & $0.123(4)$ & \\
\hline H9 & 0.8209 & -0.2788 & 0.3409 & $0.148^{*}$ & \\
\hline $\mathrm{C} 8$ & $0.7809(4)$ & -0.1765 & $0.3287(5)$ & 0.085 & \\
\hline H8 & 0.8148 & -0.1622 & 0.4055 & $0.102^{*}$ & \\
\hline $\mathrm{C} 13$ & $0.8355(3)$ & $0.0451(2)$ & $0.3297(6)$ & $0.0504(14)$ & \\
\hline $\mathrm{C} 18$ & $0.8226(3)$ & $0.1176(3)$ & $0.3135(7)$ & $0.104(4)$ & \\
\hline H18 & 0.7653 & 0.1364 & 0.2996 & $0.125^{*}$ & \\
\hline $\mathrm{C} 17$ & $0.8953(5)$ & $0.1621(2)$ & $0.3179(9)$ & $0.141(6)$ & \\
\hline H17 & 0.8867 & 0.2107 & 0.3071 & $0.169^{*}$ & \\
\hline $\mathrm{C} 16$ & $0.9809(4)$ & $0.1341(3)$ & $0.3387(8)$ & $0.115(4)$ & \\
\hline H16 & 1.0296 & 0.1639 & 0.3417 & $0.138^{*}$ & \\
\hline $\mathrm{C} 15$ & 0.9938 (3) & 0.0615 & $0.3550(7)$ & 0.092 & \\
\hline H15 & 1.0511 & 0.0428 & 0.3688 & $0.111^{*}$ & \\
\hline $\mathrm{C} 14$ & $0.9211(3)$ & $0.0170(2)$ & $0.3505(6)$ & $0.075(2)$ & \\
\hline H14 & 0.9298 & -0.0315 & 0.3614 & $0.090^{*}$ & \\
\hline C19 & $0.7557(6)$ & 0.0101 & $0.0116(7)$ & $0.0592(19)$ & \\
\hline C20B & $0.839(2)$ & $-0.0392(13)$ & $0.0291(17)$ & $0.068(6)$ & $0.66(6)$ \\
\hline
\end{tabular}




\begin{tabular}{|c|c|c|c|c|c|}
\hline $\mathrm{H} 20 \mathrm{~A}$ & 0.8263 & -0.0855 & 0.0595 & $0.081^{*}$ & $0.66(6)$ \\
\hline H20B & 0.8894 & -0.0187 & 0.0872 & $0.081 *$ & $0.66(6)$ \\
\hline $\mathrm{C} 21 \mathrm{~B}$ & $0.810(2)$ & $0.0427(14)$ & $-0.166(3)$ & $0.064(5)$ & $0.66(6)$ \\
\hline H21B & 0.7775 & 0.0400 & -0.2531 & $0.077 *$ & $0.66(6)$ \\
\hline $\mathrm{C} 22 \mathrm{~A}$ & $0.864(3)$ & $0.0842(15)$ & $-0.190(4)$ & $0.065(9)$ & $0.34(6)$ \\
\hline $\mathrm{C} 23 \mathrm{~A}$ & $0.872(4)$ & 0.1077 (19) & $-0.307(4)$ & $0.098(15)$ & $0.34(6)$ \\
\hline $\mathrm{H} 23 \mathrm{~A}$ & 0.8325 & 0.0919 & -0.3783 & $0.117 *$ & $0.34(6)$ \\
\hline $\mathrm{C} 24 \mathrm{~A}$ & $0.940(5)$ & $0.155(2)$ & $-0.317(5)$ & $0.12(2)$ & $0.34(6)$ \\
\hline $\mathrm{H} 24 \mathrm{~A}$ & 0.9452 & 0.1706 & -0.3956 & $0.147^{*}$ & $0.34(6)$ \\
\hline $\mathrm{C} 25 \mathrm{~A}$ & $0.999(4)$ & $0.179(2)$ & $-0.211(6)$ & $0.13(2)$ & $0.34(6)$ \\
\hline $\mathrm{H} 25 \mathrm{~A}$ & 1.0439 & 0.2101 & -0.2180 & $0.161 *$ & $0.34(6)$ \\
\hline $\mathrm{C} 26 \mathrm{~A}$ & $0.990(2)$ & $0.155(2)$ & $-0.094(6)$ & $0.125(15)$ & $0.34(6)$ \\
\hline $\mathrm{H} 26 \mathrm{~A}$ & 1.0300 & 0.1709 & -0.0232 & $0.150 *$ & $0.34(6)$ \\
\hline $\mathrm{C} 27 \mathrm{~A}$ & $0.923(3)$ & 0.1079 (19) & $-0.084(4)$ & $0.080(9)$ & $0.34(6)$ \\
\hline $\mathrm{H} 27 \mathrm{~A}$ & 0.9174 & 0.0922 & -0.0058 & $0.096 *$ & $0.34(6)$ \\
\hline C20A & $0.811(3)$ & -0.051 & $0.003(4)$ & $0.056(8)$ & $0.34(6)$ \\
\hline $\mathrm{H} 20 \mathrm{C}$ & 0.7807 & -0.0935 & 0.0184 & $0.067 *$ & $0.34(6)$ \\
\hline H20D & 0.8661 & -0.0471 & 0.0667 & $0.067 *$ & $0.34(6)$ \\
\hline $\mathrm{C} 21 \mathrm{~A}$ & $0.798(4)$ & $0.029(3)$ & $-0.184(6)$ & $0.060(7)$ & $0.34(6)$ \\
\hline $\mathrm{H} 21 \mathrm{~A}$ & 0.7564 & 0.0262 & -0.2650 & $0.071 *$ & $0.34(6)$ \\
\hline $\mathrm{C} 22 \mathrm{~B}$ & $0.8812(14)$ & $0.0986(13)$ & $-0.158(2)$ & $0.076(5)$ & $0.66(6)$ \\
\hline $\mathrm{C} 23 \mathrm{~B}$ & 0.9134 (19) & $0.1135(13)$ & $-0.264(3)$ & $0.103(7)$ & $0.66(6)$ \\
\hline $\mathrm{H} 23 \mathrm{~B}$ & 0.8852 & 0.0945 & -0.3402 & $0.124^{*}$ & $0.66(6)$ \\
\hline $\mathrm{C} 24 \mathrm{~B}$ & $0.988(2)$ & $0.1569(12)$ & $-0.255(4)$ & $0.132(11)$ & $0.66(6)$ \\
\hline H24B & 1.0091 & 0.1669 & -0.3264 & $0.158 *$ & $0.66(6)$ \\
\hline $\mathrm{C} 25 \mathrm{~B}$ & $1.0296(15)$ & $0.1853(13)$ & $-0.141(4)$ & $0.150(13)$ & $0.66(6)$ \\
\hline $\mathrm{H} 25 \mathrm{~B}$ & 1.0792 & 0.2143 & -0.1357 & $0.180^{*}$ & $0.66(6)$ \\
\hline $\mathrm{C} 26 \mathrm{~B}$ & 0.9975 (14) & 0.1704 (14) & -0.035 & $0.128(9)$ & $0.66(6)$ \\
\hline $\mathrm{H} 26 \mathrm{~B}$ & 1.0256 & 0.1894 & 0.0414 & $0.154^{*}$ & $0.66(6)$ \\
\hline $\mathrm{C} 27 \mathrm{~B}$ & $0.9233(15)$ & $0.1271(14)$ & $-0.043(2)$ & $0.094(6)$ & $0.66(6)$ \\
\hline $\mathrm{H} 27 \mathrm{~B}$ & 0.9018 & 0.1171 & 0.0276 & $0.113 *$ & $0.66(6)$ \\
\hline $\mathrm{C} 28$ & $0.6744(6)$ & $0.1067(4)$ & $-0.1129(7)$ & 0.0641 (19) & \\
\hline $\mathrm{H} 28$ & 0.6264 & 0.0907 & -0.0735 & $0.077 *$ & \\
\hline C29 & $0.7082(7)$ & $0.1766(5)$ & $-0.0520(10)$ & $0.093(3)$ & \\
\hline $\mathrm{H} 29 \mathrm{~A}$ & 0.7557 & 0.1948 & -0.0888 & $0.111^{*}$ & \\
\hline H29B & 0.7319 & 0.1695 & 0.0364 & $0.111^{*}$ & \\
\hline $\mathrm{C} 30$ & $0.6307(9)$ & $0.2297(6)$ & $-0.0715(11)$ & $0.120(4)$ & \\
\hline $\mathrm{H} 30 \mathrm{~A}$ & 0.5858 & 0.2131 & -0.0284 & $0.144^{*}$ & \\
\hline H30B & 0.6524 & 0.2751 & -0.0363 & $0.144^{*}$ & \\
\hline C31 & $0.5898(10)$ & $0.2386(6)$ & $-0.2066(12)$ & $0.123(4)$ & \\
\hline H31A & 0.5402 & 0.2713 & -0.2162 & $0.148 *$ & \\
\hline H31B & 0.6336 & 0.2587 & -0.2485 & $0.148 *$ & \\
\hline $\mathrm{C} 32$ & $0.5578(9)$ & $0.1698(7)$ & $-0.2659(12)$ & $0.122(4)$ & \\
\hline $\mathrm{H} 32 \mathrm{~A}$ & 0.5334 & 0.1770 & -0.3541 & $0.146^{*}$ & \\
\hline H32B & 0.5106 & 0.1514 & -0.2287 & $0.146^{*}$ & \\
\hline C33 & $0.6344(7)$ & $0.1158(5)$ & $-0.2490(8)$ & $0.086(3)$ & \\
\hline H33A & 0.6120 & 0.0707 & -0.2849 & $0.103 *$ & \\
\hline H33B & 0.6796 & 0.1323 & -0.2917 & $0.103 *$ & \\
\hline
\end{tabular}




\begin{tabular}{llllll} 
C11 & $0.74786(14)$ & $-0.04867(12)$ & $0.53404(17)$ & $0.0666(5)$ & \\
N1 & $0.7461(5)$ & $0.0513(3)$ & $-0.0871(5)$ & $0.0578(15)$ & \\
O1 & $0.7073(4)$ & $0.0135(3)$ & $0.0899(4)$ & $0.0620(13)$ & \\
S1A & $0.837(2)$ & $-0.0549(18)$ & $-0.145(3)$ & $0.067(5)$ & $0.34(6)$ \\
S1B & $0.861(2)$ & $-0.0454(11)$ & $-0.121(2)$ & $0.083(4)$ & $0.66(6)$ \\
Sn1 & $0.72177(3)$ & $-0.02084(2)$ & $0.31194(4)$ & $0.04302(17)$ & \\
\hline
\end{tabular}

Atomic displacement parameters $\left(\AA^{2}\right)$

\begin{tabular}{|c|c|c|c|c|c|c|}
\hline & $U^{11}$ & $U^{22}$ & $U^{\beta 3}$ & $U^{12}$ & $U^{13}$ & $U^{23}$ \\
\hline $\mathrm{C} 1$ & 0.037 (3) & 0.044 (3) & $0.059(4)$ & -0.008 & $0.010(3)$ & $-0.002(3)$ \\
\hline $\mathrm{C} 2$ & $0.056(5)$ & $0.162(10)$ & $0.056(4)$ & $0.029(6)$ & $0.006(4)$ & $-0.001(5)$ \\
\hline $\mathrm{C} 3$ & $0.060(6)$ & 0.167 (12) & $0.107(7)$ & $0.032(7)$ & $-0.010(5)$ & $-0.004(8)$ \\
\hline $\mathrm{C} 4$ & $0.045(5)$ & $0.077(6)$ & $0.144(8)$ & $0.018(4)$ & $0.022(4)$ & $-0.005(6)$ \\
\hline $\mathrm{C} 5$ & $0.081(7)$ & $0.103(8)$ & $0.116(7)$ & $0.023(6)$ & $0.042(5)$ & $-0.008(6)$ \\
\hline $\mathrm{C} 6$ & $0.071(5)$ & $0.099(6)$ & $0.064(5)$ & $0.027(5)$ & $0.020(4)$ & $-0.009(5)$ \\
\hline $\mathrm{C} 7$ & $0.062(5)$ & $0.050(3)$ & $0.072(4)$ & $0.005(3)$ & $0.035(4)$ & $0.006(3)$ \\
\hline $\mathrm{C} 12$ & $0.162(11)$ & $0.066(5)$ & $0.090(7)$ & -0.007 (6) & $0.011(6)$ & $-0.019(5)$ \\
\hline C11 & 0.195 (14) & $0.075(6)$ & $0.141(10)$ & $-0.037(7)$ & $0.068(8)$ & $-0.042(6)$ \\
\hline $\mathrm{C} 10$ & 0.154 (12) & $0.054(5)$ & $0.206(12)$ & $-0.025(5)$ & $0.116(9)$ & $-0.031(6)$ \\
\hline C9 & $0.132(11)$ & $0.057(5)$ & $0.201(12)$ & $0.025(6)$ & $0.080(8)$ & $0.022(6)$ \\
\hline $\mathrm{C} 8$ & $0.090(7)$ & $0.057(4)$ & $0.113(7)$ & $0.020(4)$ & $0.034(5)$ & 0.014 (4) \\
\hline $\mathrm{C} 13$ & $0.042(3)$ & $0.056(3)$ & $0.056(4)$ & $0.005(3)$ & $0.016(3)$ & $0.004(3)$ \\
\hline $\mathrm{C} 18$ & $0.061(5)$ & $0.052(4)$ & 0.199 (12) & -0.002 & $0.028(7)$ & $0.006(6)$ \\
\hline $\mathrm{C} 17$ & $0.083(7)$ & $0.074(6)$ & 0.257 (17) & $-0.023(5)$ & $0.016(9)$ & $0.024(9)$ \\
\hline $\mathrm{C} 16$ & $0.071(5)$ & $0.104(6)$ & $0.173(12)$ & $-0.034(5)$ & $0.034(7)$ & $0.011(8)$ \\
\hline C15 & $0.054(5)$ & $0.115(7)$ & $0.117(8)$ & $-0.004(5)$ & $0.037(5)$ & 0.009 (7) \\
\hline C14 & $0.051(4)$ & $0.078(5)$ & $0.098(7)$ & $0.005(4)$ & 0.019 (4) & $0.002(5)$ \\
\hline C19 & $0.086(6)$ & $0.055(4)$ & $0.042(3)$ & 0.008 (3) & $0.025(3)$ & $0.006(3)$ \\
\hline $\mathrm{C} 20 \mathrm{~B}$ & $0.112(15)$ & $0.075(9)$ & $0.016(6)$ & $0.041(10)$ & $0.015(8)$ & $-0.020(5)$ \\
\hline $\mathrm{C} 21 \mathrm{~B}$ & $0.114(12)$ & $0.046(9)$ & $0.041(8)$ & $0.014(7)$ & $0.038(8)$ & $-0.006(6)$ \\
\hline $\mathrm{C} 22 \mathrm{~A}$ & $0.09(2)$ & 0.037 (10) & $0.082(18)$ & $0.008(12)$ & 0.054 (16) & $-0.017(11)$ \\
\hline $\mathrm{C} 23 \mathrm{~A}$ & $0.16(4)$ & 0.048 (16) & $0.12(2)$ & $-0.01(2)$ & $0.10(2)$ & $-0.001(17)$ \\
\hline $\mathrm{C} 24 \mathrm{~A}$ & $0.14(5)$ & $0.06(2)$ & $0.21(4)$ & $0.00(3)$ & $0.13(4)$ & $0.02(3)$ \\
\hline $\mathrm{C} 25 \mathrm{~A}$ & $0.12(3)$ & 0.043 (19) & $0.28(5)$ & $0.00(2)$ & $0.13(4)$ & $-0.04(3)$ \\
\hline $\mathrm{C} 26 \mathrm{~A}$ & $0.05(2)$ & $0.09(3)$ & $0.23(4)$ & $0.015(14)$ & $0.02(2)$ & $-0.01(3)$ \\
\hline C27A & 0.053 (16) & $0.050(17)$ & $0.14(2)$ & $0.033(12)$ & 0.017 (17) & $-0.008(19)$ \\
\hline $\mathrm{C} 20 \mathrm{~A}$ & 0.060 (18) & $0.080(17)$ & $0.021(13)$ & $0.016(13)$ & $-0.003(12)$ & $0.016(13)$ \\
\hline $\mathrm{C} 21 \mathrm{~A}$ & $0.10(2)$ & $0.042(15)$ & $0.050(17)$ & $0.004(10)$ & $0.037(15)$ & $0.011(14)$ \\
\hline $\mathrm{C} 22 \mathrm{~B}$ & 0.079 (11) & $0.069(9)$ & 0.093 (12) & $0.024(9)$ & $0.047(9)$ & $0.015(9)$ \\
\hline $\mathrm{C} 23 \mathrm{~B}$ & 0.112 (17) & 0.082 (14) & $0.142(15)$ & $0.032(10)$ & $0.087(14)$ & $0.025(12)$ \\
\hline C24B & $0.11(2)$ & $0.066(14)$ & $0.25(3)$ & $0.041(13)$ & $0.12(2)$ & 0.047 (17) \\
\hline $\mathrm{C} 25 \mathrm{~B}$ & $0.081(15)$ & 0.085 (19) & $0.29(4)$ & $0.027(10)$ & 0.057 (18) & $0.05(2)$ \\
\hline $\mathrm{C} 26 \mathrm{~B}$ & $0.071(12)$ & 0.079 (14) & $0.22(2)$ & $0.021(9)$ & $0.012(14)$ & $0.015(15)$ \\
\hline C27B & $0.076(11)$ & 0.070 (14) & $0.136(14)$ & $0.018(9)$ & $0.021(10)$ & $-0.015(11)$ \\
\hline $\mathrm{C} 28$ & $0.084(6)$ & 0.060 (4) & 0.052 (4) & $0.015(4)$ & 0.023 (4) & 0.005 (3) \\
\hline C29 & 0.099 (7) & $0.072(5)$ & 0.099 (7) & $0.022(5)$ & $0.006(5)$ & $-0.027(5)$ \\
\hline C30 & $0.157(11)$ & $0.084(7)$ & $0.116(7)$ & $0.055(7)$ & $0.021(7)$ & $-0.013(6)$ \\
\hline
\end{tabular}




\begin{tabular}{lllllll} 
C31 & $0.145(11)$ & $0.097(7)$ & $0.126(8)$ & $0.057(7)$ & $0.024(7)$ & $0.017(7)$ \\
C32 & $0.116(10)$ & $0.124(8)$ & $0.108(8)$ & $0.039(7)$ & $-0.013(7)$ & $0.014(6)$ \\
C33 & $0.106(8)$ & $0.078(6)$ & $0.065(5)$ & $0.011(5)$ & $0.002(5)$ & $0.003(4)$ \\
C11 & $0.0649(12)$ & $0.0897(14)$ & $0.0434(9)$ & $-0.0031(10)$ & $0.0077(8)$ & $0.0144(9)$ \\
N1 & $0.080(4)$ & $0.056(3)$ & $0.044(3)$ & $0.015(3)$ & $0.026(3)$ & $0.005(2)$ \\
O1 & $0.082(4)$ & $0.074(3)$ & $0.034(2)$ & $0.011(3)$ & $0.020(2)$ & $0.006(2)$ \\
S1A & $0.097(12)$ & $0.051(7)$ & $0.057(9)$ & $0.020(6)$ & $0.030(7)$ & $0.003(6)$ \\
S1B & $0.137(12)$ & $0.062(5)$ & $0.064(6)$ & $0.028(6)$ & $0.050(7)$ & $0.001(4)$ \\
Sn1 & $0.0411(3)$ & $0.0463(3)$ & $0.0426(3)$ & $0.0028(2)$ & $0.01113(18)$ & $0.00347(19)$ \\
\hline
\end{tabular}

Geometric parameters $\left(\AA,{ }^{\circ}\right)$

\begin{tabular}{|c|c|c|c|}
\hline $\mathrm{Sn} 1-\mathrm{C} 1$ & $2.141(4)$ & $\mathrm{C} 21 \mathrm{~B}-\mathrm{S} 1 \mathrm{~B}$ & $1.86(4)$ \\
\hline $\mathrm{Sn} 1-\mathrm{C} 7$ & $2.130(4)$ & $\mathrm{C} 22 \mathrm{~A}-\mathrm{C} 23 \mathrm{~A}$ & 1.3900 \\
\hline $\mathrm{Sn} 1-\mathrm{C} 13$ & $2.119(4)$ & $\mathrm{C} 22 \mathrm{~A}-\mathrm{C} 27 \mathrm{~A}$ & 1.3900 \\
\hline Sn1-Cl1 & $2.4439(19)$ & $\mathrm{C} 22 \mathrm{~A}-\mathrm{C} 21 \mathrm{~A}$ & $1.47(4)$ \\
\hline $\mathrm{Sn} 1-\mathrm{O} 1$ & $2.488(4)$ & $\mathrm{C} 23 \mathrm{~A}-\mathrm{H} 23 \mathrm{~A}$ & 0.9300 \\
\hline $\mathrm{C} 1-\mathrm{C} 2$ & 1.3900 & $\mathrm{C} 23 \mathrm{~A}-\mathrm{C} 24 \mathrm{~A}$ & 1.3900 \\
\hline $\mathrm{C} 1-\mathrm{C} 6$ & 1.3900 & $\mathrm{C} 24 \mathrm{~A}-\mathrm{H} 24 \mathrm{~A}$ & 0.9300 \\
\hline $\mathrm{C} 2-\mathrm{H} 2$ & 0.9300 & $\mathrm{C} 24 \mathrm{~A}-\mathrm{C} 25 \mathrm{~A}$ & 1.3900 \\
\hline $\mathrm{C} 2-\mathrm{C} 3$ & 1.3900 & $\mathrm{C} 25 \mathrm{~A}-\mathrm{H} 25 \mathrm{~A}$ & 0.9300 \\
\hline $\mathrm{C} 3-\mathrm{H} 3$ & 0.9300 & $\mathrm{C} 25 \mathrm{~A}-\mathrm{C} 26 \mathrm{~A}$ & 1.3900 \\
\hline $\mathrm{C} 3-\mathrm{C} 4$ & 1.3900 & $\mathrm{C} 26 \mathrm{~A}-\mathrm{H} 26 \mathrm{~A}$ & 0.9300 \\
\hline $\mathrm{C} 4-\mathrm{H} 4$ & 0.9300 & $\mathrm{C} 26 \mathrm{~A}-\mathrm{C} 27 \mathrm{~A}$ & 1.3900 \\
\hline $\mathrm{C} 4-\mathrm{C} 5$ & 1.3900 & $\mathrm{C} 27 \mathrm{~A}-\mathrm{H} 27 \mathrm{~A}$ & 0.9300 \\
\hline $\mathrm{C} 5-\mathrm{H} 5$ & 0.9300 & $\mathrm{C} 20 \mathrm{~A}-\mathrm{H} 20 \mathrm{C}$ & 0.9700 \\
\hline $\mathrm{C} 5-\mathrm{C} 6$ & 1.3900 & $\mathrm{C} 20 \mathrm{~A}-\mathrm{H} 20 \mathrm{D}$ & 0.9700 \\
\hline $\mathrm{C} 6-\mathrm{H} 6$ & 0.9300 & $\mathrm{C} 20 \mathrm{~A}-\mathrm{S} 1 \mathrm{~A}$ & $1.76(5)$ \\
\hline $\mathrm{C} 7-\mathrm{C} 12$ & 1.3900 & $\mathrm{C} 21 \mathrm{~A}-\mathrm{H} 21 \mathrm{~A}$ & 0.9800 \\
\hline $\mathrm{C} 7-\mathrm{C} 8$ & 1.3900 & $\mathrm{C} 21 \mathrm{~A}-\mathrm{N} 1$ & $1.52(6)$ \\
\hline $\mathrm{C} 12-\mathrm{H} 12$ & 0.9300 & $\mathrm{C} 21 \mathrm{~A}-\mathrm{S} 1 \mathrm{~A}$ & $1.71(7)$ \\
\hline $\mathrm{C} 12-\mathrm{C} 11$ & 1.3900 & $\mathrm{C} 22 \mathrm{~B}-\mathrm{C} 23 \mathrm{~B}$ & 1.3900 \\
\hline C11-H11 & 0.9300 & $\mathrm{C} 22 \mathrm{~B}-\mathrm{C} 27 \mathrm{~B}$ & 1.3900 \\
\hline $\mathrm{C} 11-\mathrm{C} 10$ & 1.3900 & $\mathrm{C} 23 \mathrm{~B}-\mathrm{H} 23 \mathrm{~B}$ & 0.9300 \\
\hline $\mathrm{C} 10-\mathrm{H} 10$ & 0.9300 & $\mathrm{C} 23 \mathrm{~B}-\mathrm{C} 24 \mathrm{~B}$ & 1.3900 \\
\hline $\mathrm{C} 10-\mathrm{C} 9$ & 1.3900 & $\mathrm{C} 24 \mathrm{~B}-\mathrm{H} 24 \mathrm{~B}$ & 0.9300 \\
\hline C9- $\mathrm{H} 9$ & 0.9300 & $\mathrm{C} 24 \mathrm{~B}-\mathrm{C} 25 \mathrm{~B}$ & 1.3900 \\
\hline $\mathrm{C} 9-\mathrm{C} 8$ & 1.3900 & $\mathrm{C} 25 \mathrm{~B}-\mathrm{H} 25 \mathrm{~B}$ & 0.9300 \\
\hline $\mathrm{C} 8-\mathrm{H} 8$ & 0.9300 & $\mathrm{C} 25 \mathrm{~B}-\mathrm{C} 26 \mathrm{~B}$ & 1.3900 \\
\hline $\mathrm{C} 13-\mathrm{C} 18$ & 1.3900 & $\mathrm{C} 26 \mathrm{~B}-\mathrm{H} 26 \mathrm{~B}$ & 0.9300 \\
\hline $\mathrm{C} 13-\mathrm{C} 14$ & 1.3900 & $\mathrm{C} 26 \mathrm{~B}-\mathrm{C} 27 \mathrm{~B}$ & 1.3900 \\
\hline $\mathrm{C} 18-\mathrm{H} 18$ & 0.9300 & $\mathrm{C} 27 \mathrm{~B}-\mathrm{H} 27 \mathrm{~B}$ & 0.9300 \\
\hline $\mathrm{C} 18-\mathrm{C} 17$ & 1.3900 & $\mathrm{C} 28-\mathrm{H} 28$ & 0.9800 \\
\hline C17-H17 & 0.9300 & $\mathrm{C} 28-\mathrm{C} 29$ & $1.519(12)$ \\
\hline $\mathrm{C} 17-\mathrm{C} 16$ & 1.3900 & $\mathrm{C} 28-\mathrm{C} 33$ & 1.499 (11) \\
\hline $\mathrm{C} 16-\mathrm{H} 16$ & 0.9300 & $\mathrm{C} 28-\mathrm{N} 1$ & $1.501(10)$ \\
\hline $\mathrm{C} 16-\mathrm{C} 15$ & 1.3900 & $\mathrm{C} 29-\mathrm{H} 29 \mathrm{~A}$ & 0.9700 \\
\hline C15-H15 & 0.9300 & $\mathrm{C} 29-\mathrm{H} 29 \mathrm{~B}$ & 0.9700 \\
\hline
\end{tabular}




\begin{tabular}{|c|c|c|c|}
\hline $\mathrm{C} 15-\mathrm{C} 14$ & 1.3900 & $\mathrm{C} 29-\mathrm{C} 30$ & $1.536(13)$ \\
\hline $\mathrm{C} 14-\mathrm{H} 14$ & 0.9300 & $\mathrm{C} 30-\mathrm{H} 30 \mathrm{~A}$ & 0.9700 \\
\hline $\mathrm{C} 19-\mathrm{C} 20 \mathrm{~B}$ & $1.56(3)$ & $\mathrm{C} 30-\mathrm{H} 30 \mathrm{~B}$ & 0.9700 \\
\hline $\mathrm{C} 19-\mathrm{C} 20 \mathrm{~A}$ & $1.44(4)$ & $\mathrm{C} 30-\mathrm{C} 31$ & $1.491(15)$ \\
\hline $\mathrm{C} 19-\mathrm{N} 1$ & $1.317(9)$ & $\mathrm{C} 31-\mathrm{H} 31 \mathrm{~A}$ & 0.9700 \\
\hline $\mathrm{C} 19-\mathrm{O} 1$ & $1.257(8)$ & $\mathrm{C} 31-\mathrm{H} 31 \mathrm{~B}$ & 0.9700 \\
\hline $\mathrm{C} 20 \mathrm{~B}-\mathrm{H} 20 \mathrm{~A}$ & 0.9700 & $\mathrm{C} 31-\mathrm{C} 32$ & $1.488(17)$ \\
\hline $\mathrm{C} 20 \mathrm{~B}-\mathrm{H} 20 \mathrm{~B}$ & 0.9700 & $\mathrm{C} 32-\mathrm{H} 32 \mathrm{~A}$ & 0.9700 \\
\hline $\mathrm{C} 20 \mathrm{~B}-\mathrm{S} 1 \mathrm{~B}$ & $1.76(2)$ & $\mathrm{C} 32-\mathrm{H} 32 \mathrm{~B}$ & 0.9700 \\
\hline $\mathrm{C} 21 \mathrm{~B}-\mathrm{H} 21 \mathrm{~B}$ & 0.9800 & $\mathrm{C} 32-\mathrm{C} 33$ & $1.537(14)$ \\
\hline $\mathrm{C} 21 \mathrm{~B}-\mathrm{C} 22 \mathrm{~B}$ & $1.50(2)$ & $\mathrm{C} 33-\mathrm{H} 33 \mathrm{~A}$ & 0.9700 \\
\hline $\mathrm{C} 21 \mathrm{~B}-\mathrm{N} 1$ & $1.46(3)$ & С $33-\mathrm{H} 33 \mathrm{~B}$ & 0.9700 \\
\hline $\mathrm{C} 2-\mathrm{C} 1-\mathrm{C} 6$ & 120.0 & $\mathrm{C} 26 \mathrm{~A}-\mathrm{C} 27 \mathrm{~A}-\mathrm{C} 22 \mathrm{~A}$ & 120.0 \\
\hline $\mathrm{C} 2-\mathrm{C} 1-\mathrm{Sn} 1$ & $121.3(3)$ & $\mathrm{C} 26 \mathrm{~A}-\mathrm{C} 27 \mathrm{~A}-\mathrm{H} 27 \mathrm{~A}$ & 120.0 \\
\hline $\mathrm{C} 6-\mathrm{C} 1-\mathrm{Sn} 1$ & $118.7(3)$ & $\mathrm{C} 19-\mathrm{C} 20 \mathrm{~A}-\mathrm{H} 20 \mathrm{C}$ & 109.5 \\
\hline $\mathrm{C} 1-\mathrm{C} 2-\mathrm{H} 2$ & 120.0 & $\mathrm{C} 19-\mathrm{C} 20 \mathrm{~A}-\mathrm{H} 20 \mathrm{D}$ & 109.5 \\
\hline $\mathrm{C} 1-\mathrm{C} 2-\mathrm{C} 3$ & 120.0 & $\mathrm{C} 19-\mathrm{C} 20 \mathrm{~A}-\mathrm{S} 1 \mathrm{~A}$ & $111(2)$ \\
\hline $\mathrm{C} 3-\mathrm{C} 2-\mathrm{H} 2$ & 120.0 & $\mathrm{H} 20 \mathrm{C}-\mathrm{C} 20 \mathrm{~A}-\mathrm{H} 20 \mathrm{D}$ & 108.1 \\
\hline $\mathrm{C} 2-\mathrm{C} 3-\mathrm{H} 3$ & 120.0 & $\mathrm{~S} 1 \mathrm{~A}-\mathrm{C} 20 \mathrm{~A}-\mathrm{H} 20 \mathrm{C}$ & 109.5 \\
\hline $\mathrm{C} 2-\mathrm{C} 3-\mathrm{C} 4$ & 120.0 & $\mathrm{~S} 1 \mathrm{~A}-\mathrm{C} 20 \mathrm{~A}-\mathrm{H} 20 \mathrm{D}$ & 109.5 \\
\hline $\mathrm{C} 4-\mathrm{C} 3-\mathrm{H} 3$ & 120.0 & $\mathrm{C} 22 \mathrm{~A}-\mathrm{C} 21 \mathrm{~A}-\mathrm{H} 21 \mathrm{~A}$ & 108.1 \\
\hline $\mathrm{C} 3-\mathrm{C} 4-\mathrm{H} 4$ & 120.0 & $\mathrm{C} 22 \mathrm{~A}-\mathrm{C} 21 \mathrm{~A}-\mathrm{N} 1$ & $108(4)$ \\
\hline $\mathrm{C} 5-\mathrm{C} 4-\mathrm{C} 3$ & 120.0 & $\mathrm{C} 22 \mathrm{~A}-\mathrm{C} 21 \mathrm{~A}-\mathrm{S} 1 \mathrm{~A}$ & $118(4)$ \\
\hline $\mathrm{C} 5-\mathrm{C} 4-\mathrm{H} 4$ & 120.0 & $\mathrm{~N} 1-\mathrm{C} 21 \mathrm{~A}-\mathrm{H} 21 \mathrm{~A}$ & 108.1 \\
\hline $\mathrm{C} 4-\mathrm{C} 5-\mathrm{H} 5$ & 120.0 & $\mathrm{~N} 1-\mathrm{C} 21 \mathrm{~A}-\mathrm{S} 1 \mathrm{~A}$ & $107(3)$ \\
\hline $\mathrm{C} 6-\mathrm{C} 5-\mathrm{C} 4$ & 120.0 & $\mathrm{~S} 1 \mathrm{~A}-\mathrm{C} 21 \mathrm{~A}-\mathrm{H} 21 \mathrm{~A}$ & 108.1 \\
\hline $\mathrm{C} 6-\mathrm{C} 5-\mathrm{H} 5$ & 120.0 & $\mathrm{C} 23 \mathrm{~B}-\mathrm{C} 22 \mathrm{~B}-\mathrm{C} 21 \mathrm{~B}$ & $118.2(12)$ \\
\hline $\mathrm{C} 1-\mathrm{C} 6-\mathrm{H} 6$ & 120.0 & $\mathrm{C} 23 \mathrm{~B}-\mathrm{C} 22 \mathrm{~B}-\mathrm{C} 27 \mathrm{~B}$ & 120.0 \\
\hline $\mathrm{C} 5-\mathrm{C} 6-\mathrm{C} 1$ & 120.0 & $\mathrm{C} 27 \mathrm{~B}-\mathrm{C} 22 \mathrm{~B}-\mathrm{C} 21 \mathrm{~B}$ & $121.1(12)$ \\
\hline $\mathrm{C} 5-\mathrm{C} 6-\mathrm{H} 6$ & 120.0 & $\mathrm{C} 22 \mathrm{~B}-\mathrm{C} 23 \mathrm{~B}-\mathrm{H} 23 \mathrm{~B}$ & 120.0 \\
\hline $\mathrm{C} 12-\mathrm{C} 7-\mathrm{C} 8$ & 120.0 & $\mathrm{C} 22 \mathrm{~B}-\mathrm{C} 23 \mathrm{~B}-\mathrm{C} 24 \mathrm{~B}$ & 120.0 \\
\hline $\mathrm{C} 12-\mathrm{C} 7-\mathrm{Sn} 1$ & $120.1(3)$ & $\mathrm{C} 24 \mathrm{~B}-\mathrm{C} 23 \mathrm{~B}-\mathrm{H} 23 \mathrm{~B}$ & 120.0 \\
\hline $\mathrm{C} 8-\mathrm{C} 7-\mathrm{Sn} 1$ & $119.9(3)$ & $\mathrm{C} 23 \mathrm{~B}-\mathrm{C} 24 \mathrm{~B}-\mathrm{H} 24 \mathrm{~B}$ & 120.0 \\
\hline $\mathrm{C} 7-\mathrm{C} 12-\mathrm{H} 12$ & 120.0 & $\mathrm{C} 23 \mathrm{~B}-\mathrm{C} 24 \mathrm{~B}-\mathrm{C} 25 \mathrm{~B}$ & 120.0 \\
\hline $\mathrm{C} 11-\mathrm{C} 12-\mathrm{C} 7$ & 120.0 & $\mathrm{C} 25 \mathrm{~B}-\mathrm{C} 24 \mathrm{~B}-\mathrm{H} 24 \mathrm{~B}$ & 120.0 \\
\hline $\mathrm{C} 11-\mathrm{C} 12-\mathrm{H} 12$ & 120.0 & $\mathrm{C} 24 \mathrm{~B}-\mathrm{C} 25 \mathrm{~B}-\mathrm{H} 25 \mathrm{~B}$ & 120.0 \\
\hline $\mathrm{C} 12-\mathrm{C} 11-\mathrm{H} 11$ & 120.0 & $\mathrm{C} 26 \mathrm{~B}-\mathrm{C} 25 \mathrm{~B}-\mathrm{C} 24 \mathrm{~B}$ & 120.0 \\
\hline $\mathrm{C} 12-\mathrm{C} 11-\mathrm{C} 10$ & 120.0 & $\mathrm{C} 26 \mathrm{~B}-\mathrm{C} 25 \mathrm{~B}-\mathrm{H} 25 \mathrm{~B}$ & 120.0 \\
\hline $\mathrm{C} 10-\mathrm{C} 11-\mathrm{H} 11$ & 120.0 & $\mathrm{C} 25 \mathrm{~B}-\mathrm{C} 26 \mathrm{~B}-\mathrm{H} 26 \mathrm{~B}$ & 120.0 \\
\hline $\mathrm{C} 11-\mathrm{C} 10-\mathrm{H} 10$ & 120.0 & $\mathrm{C} 25 \mathrm{~B}-\mathrm{C} 26 \mathrm{~B}-\mathrm{C} 27 \mathrm{~B}$ & 120.0 \\
\hline $\mathrm{C} 9-\mathrm{C} 10-\mathrm{C} 11$ & 120.0 & $\mathrm{C} 27 \mathrm{~B}-\mathrm{C} 26 \mathrm{~B}-\mathrm{H} 26 \mathrm{~B}$ & 120.0 \\
\hline $\mathrm{C} 9-\mathrm{C} 10-\mathrm{H} 10$ & 120.0 & $\mathrm{C} 22 \mathrm{~B}-\mathrm{C} 27 \mathrm{~B}-\mathrm{H} 27 \mathrm{~B}$ & 120.0 \\
\hline $\mathrm{C} 10-\mathrm{C} 9-\mathrm{H} 9$ & 120.0 & $\mathrm{C} 26 \mathrm{~B}-\mathrm{C} 27 \mathrm{~B}-\mathrm{C} 22 \mathrm{~B}$ & 120.0 \\
\hline $\mathrm{C} 10-\mathrm{C} 9-\mathrm{C} 8$ & 120.0 & $\mathrm{C} 26 \mathrm{~B}-\mathrm{C} 27 \mathrm{~B}-\mathrm{H} 27 \mathrm{~B}$ & 120.0 \\
\hline $\mathrm{C} 8-\mathrm{C} 9-\mathrm{H} 9$ & 120.0 & $\mathrm{C} 29-\mathrm{C} 28-\mathrm{H} 28$ & 107.0 \\
\hline $\mathrm{C} 7-\mathrm{C} 8-\mathrm{H} 8$ & 120.0 & $\mathrm{C} 33-\mathrm{C} 28-\mathrm{H} 28$ & 107.0 \\
\hline $\mathrm{C} 9-\mathrm{C} 8-\mathrm{C} 7$ & 120.0 & $\mathrm{C} 33-\mathrm{C} 28-\mathrm{C} 29$ & $111.6(7)$ \\
\hline
\end{tabular}


C9- 8 - $-\mathrm{H} 8$

$\mathrm{C} 18-\mathrm{C} 13-\mathrm{C} 14$

$\mathrm{C} 18-\mathrm{C} 13-\mathrm{Sn} 1$

$\mathrm{C} 14-\mathrm{C} 13-\mathrm{Sn} 1$

C13-C18-H18

C13-C18-C17

C17-C18-H18

C18-C17-H17

C16-C17-C18

$\mathrm{C} 16-\mathrm{C} 17-\mathrm{H} 17$

$\mathrm{C} 17-\mathrm{C} 16-\mathrm{H} 16$

$\mathrm{C} 17-\mathrm{C} 16-\mathrm{C} 15$

$\mathrm{C} 15-\mathrm{C} 16-\mathrm{H} 16$

$\mathrm{C} 16-\mathrm{C} 15-\mathrm{H} 15$

$\mathrm{C} 14-\mathrm{C} 15-\mathrm{C} 16$

C14-C15-H15

C13-C14-H14

$\mathrm{C} 15-\mathrm{C} 14-\mathrm{C} 13$

$\mathrm{C} 15-\mathrm{C} 14-\mathrm{H} 14$

$\mathrm{C} 20 \mathrm{~A}-\mathrm{C} 19-\mathrm{C} 20 \mathrm{~B}$

N1-C19-C20B

$\mathrm{N} 1-\mathrm{C} 19-\mathrm{C} 20 \mathrm{~A}$

$\mathrm{O} 1-\mathrm{C} 19-\mathrm{C} 20 \mathrm{~B}$

O1-C19-C20A

$\mathrm{O} 1-\mathrm{C} 19-\mathrm{N} 1$

C19-C20B-H20A

$\mathrm{C} 19-\mathrm{C} 20 \mathrm{~B}-\mathrm{H} 20 \mathrm{~B}$

C19-C20B-S1B

$\mathrm{H} 20 \mathrm{~A}-\mathrm{C} 20 \mathrm{~B}-\mathrm{H} 20 \mathrm{~B}$

$\mathrm{S} 1 \mathrm{~B}-\mathrm{C} 20 \mathrm{~B}-\mathrm{H} 20 \mathrm{~A}$

$\mathrm{S} 1 \mathrm{~B}-\mathrm{C} 20 \mathrm{~B}-\mathrm{H} 20 \mathrm{~B}$

$\mathrm{C} 22 \mathrm{~B}-\mathrm{C} 21 \mathrm{~B}-\mathrm{H} 21 \mathrm{~B}$

$\mathrm{C} 22 \mathrm{~B}-\mathrm{C} 21 \mathrm{~B}-\mathrm{S} 1 \mathrm{~B}$

$\mathrm{N} 1-\mathrm{C} 21 \mathrm{~B}-\mathrm{H} 21 \mathrm{~B}$

$\mathrm{N} 1-\mathrm{C} 21 \mathrm{~B}-\mathrm{C} 22 \mathrm{~B}$

N1-C21B-S1B

$\mathrm{S} 1 \mathrm{~B}-\mathrm{C} 21 \mathrm{~B}-\mathrm{H} 21 \mathrm{~B}$

$\mathrm{C} 23 \mathrm{~A}-\mathrm{C} 22 \mathrm{~A}-\mathrm{C} 27 \mathrm{~A}$

$\mathrm{C} 23 \mathrm{~A}-\mathrm{C} 22 \mathrm{~A}-\mathrm{C} 21 \mathrm{~A}$

$\mathrm{C} 27 \mathrm{~A}-\mathrm{C} 22 \mathrm{~A}-\mathrm{C} 21 \mathrm{~A}$

$\mathrm{C} 22 \mathrm{~A}-\mathrm{C} 23 \mathrm{~A}-\mathrm{H} 23 \mathrm{~A}$

$\mathrm{C} 24 \mathrm{~A}-\mathrm{C} 23 \mathrm{~A}-\mathrm{C} 22 \mathrm{~A}$

$\mathrm{C} 24 \mathrm{~A}-\mathrm{C} 23 \mathrm{~A}-\mathrm{H} 23 \mathrm{~A}$

$\mathrm{C} 23 \mathrm{~A}-\mathrm{C} 24 \mathrm{~A}-\mathrm{H} 24 \mathrm{~A}$

$\mathrm{C} 25 \mathrm{~A}-\mathrm{C} 24 \mathrm{~A}-\mathrm{C} 23 \mathrm{~A}$

$\mathrm{C} 25 \mathrm{~A}-\mathrm{C} 24 \mathrm{~A}-\mathrm{H} 24 \mathrm{~A}$

$\mathrm{C} 24 \mathrm{~A}-\mathrm{C} 25 \mathrm{~A}-\mathrm{H} 25 \mathrm{~A}$

$\mathrm{C} 24 \mathrm{~A}-\mathrm{C} 25 \mathrm{~A}-\mathrm{C} 26 \mathrm{~A}$
120.0

120.0

$118.4(3)$

$121.5(3)$

120.0

120.0

120.0

120.0

120.0

120.0

120.0

120.0

120.0

120.0

120.0

120.0

120.0

120.0

120.0

19 (2)

$113.4(10)$

$112.3(16)$

$122.6(10)$

$122.1(17)$

$123.8(7)$

111.0

111.0

104.0 (13)

109.0

111.0

111.0

108.2

111 (2)

108.2

$117.3(17)$

$103.6(16)$

108.2

120.0

118 (2)

$121(2)$

120.0

120.0

120.0

120.0

120.0

120.0

120.0

120.0
$\mathrm{C} 33-\mathrm{C} 28-\mathrm{N} 1$

$\mathrm{N} 1-\mathrm{C} 28-\mathrm{H} 28$

$\mathrm{N} 1-\mathrm{C} 28-\mathrm{C} 29$

$\mathrm{C} 28-\mathrm{C} 29-\mathrm{H} 29 \mathrm{~A}$

C28-C29-H29B

$\mathrm{C} 28-\mathrm{C} 29-\mathrm{C} 30$

$\mathrm{H} 29 \mathrm{~A}-\mathrm{C} 29-\mathrm{H} 29 \mathrm{~B}$

C30-C29-H29A

C $30-\mathrm{C} 29-\mathrm{H} 29 \mathrm{~B}$

$\mathrm{C} 29-\mathrm{C} 30-\mathrm{H} 30 \mathrm{~A}$

C29- $\mathrm{C} 30-\mathrm{H} 30 \mathrm{~B}$

$\mathrm{H} 30 \mathrm{~A}-\mathrm{C} 30-\mathrm{H} 30 \mathrm{~B}$

$\mathrm{C} 31-\mathrm{C} 30-\mathrm{C} 29$

$\mathrm{C} 31-\mathrm{C} 30-\mathrm{H} 30 \mathrm{~A}$

C $31-\mathrm{C} 30-\mathrm{H} 30 \mathrm{~B}$

C $30-\mathrm{C} 31-\mathrm{H} 31 \mathrm{~A}$

C30-C31-H31B

H31A-C31-H31B

$\mathrm{C} 32-\mathrm{C} 31-\mathrm{C} 30$

$\mathrm{C} 32-\mathrm{C} 31-\mathrm{H} 31 \mathrm{~A}$

C32-C $31-\mathrm{H} 31 \mathrm{~B}$

$\mathrm{C} 31-\mathrm{C} 32-\mathrm{H} 32 \mathrm{~A}$

C $31-\mathrm{C} 32-\mathrm{H} 32 \mathrm{~B}$

$\mathrm{C} 31-\mathrm{C} 32-\mathrm{C} 33$

$\mathrm{H} 32 \mathrm{~A}-\mathrm{C} 32-\mathrm{H} 32 \mathrm{~B}$

C $33-\mathrm{C} 32-\mathrm{H} 32 \mathrm{~A}$

C $33-\mathrm{C} 32-\mathrm{H} 32 \mathrm{~B}$

$\mathrm{C} 28-\mathrm{C} 33-\mathrm{C} 32$

C28-C $33-\mathrm{H} 33 \mathrm{~A}$

C28-C $33-\mathrm{H} 33 \mathrm{~B}$

C $32-\mathrm{C} 33-\mathrm{H} 33 \mathrm{~A}$

C32-C $33-\mathrm{H} 33 \mathrm{~B}$

$\mathrm{H} 33 \mathrm{~A}-\mathrm{C} 33-\mathrm{H} 33 \mathrm{~B}$

C19-N1-C21B

C19-N1-C21A

C19-N1-C28

C21B-N1-C21A

$\mathrm{C} 21 \mathrm{~B}-\mathrm{N} 1-\mathrm{C} 28$

$\mathrm{C} 28-\mathrm{N} 1-\mathrm{C} 21 \mathrm{~A}$

C19-O1-Sn1

$\mathrm{C} 21 \mathrm{~A}-\mathrm{S} 1 \mathrm{~A}-\mathrm{C} 20 \mathrm{~A}$

C20B-S1B-C21B

C1-Sn1-Cl1

C1-Sn1-O1

C7-Sn1-C1

C7-Sn1-C11

C7-Sn1-O1

C13-Sn1-C1
$113.1(6)$

107.0

$110.8(7)$

109.9

109.9

109.0 (9)

108.3

109.9

109.9

109.4

109.4

108.0

$111.0(9)$

109.4

109.4

109.3

109.3

108.0

111.5 (10)

109.3

109.3

109.5

109.5

110.7 (10)

108.1

109.5

109.5

109.5 (8)

109.8

109.8

109.8

109.8

108.2

117.1 (14)

115 (2)

120.9 (6)

14 (3)

121.9 (13)

123 (2)

135.9 (5)

93 (3)

92.3 (14)

98.31 (14)

84.26 (18)

118.5 (2)

95.29 (16)

87.11 (19)

118.0 (2) 
$\mathrm{C} 26 \mathrm{~A}-\mathrm{C} 25 \mathrm{~A}-\mathrm{H} 25 \mathrm{~A}$

$\mathrm{C} 25 \mathrm{~A}-\mathrm{C} 26 \mathrm{~A}-\mathrm{H} 26 \mathrm{~A}$

$\mathrm{C} 25 \mathrm{~A}-\mathrm{C} 26 \mathrm{~A}-\mathrm{C} 27 \mathrm{~A}$

$\mathrm{C} 27 \mathrm{~A}-\mathrm{C} 26 \mathrm{~A}-\mathrm{H} 26 \mathrm{~A}$

$\mathrm{C} 22 \mathrm{~A}-\mathrm{C} 27 \mathrm{~A}-\mathrm{H} 27 \mathrm{~A}$

$\mathrm{C} 1-\mathrm{C} 2-\mathrm{C} 3-\mathrm{C} 4$

$\mathrm{C} 2-\mathrm{C} 1-\mathrm{C} 6-\mathrm{C} 5$

$\mathrm{C} 2-\mathrm{C} 1-\mathrm{Sn} 1-\mathrm{C} 7$

$\mathrm{C} 2-\mathrm{C} 1-\mathrm{Sn} 1-\mathrm{C} 13$

$\mathrm{C} 2-\mathrm{C} 1-\mathrm{Sn} 1-\mathrm{C} 1$

$\mathrm{C} 2-\mathrm{C} 1-\mathrm{Sn} 1-\mathrm{O} 1$

$\mathrm{C} 2-\mathrm{C} 3-\mathrm{C} 4-\mathrm{C} 5$

$\mathrm{C} 3-\mathrm{C} 4-\mathrm{C} 5-\mathrm{C} 6$

$\mathrm{C} 4-\mathrm{C} 5-\mathrm{C} 6-\mathrm{C} 1$

$\mathrm{C} 6-\mathrm{C} 1-\mathrm{C} 2-\mathrm{C} 3$

C6- 1 1-Sn1-C7

C6- C1-Sn1-C13

C6- 1 1-Sn1-Cl1

C6- 1 - $\mathrm{Sn} 1-\mathrm{O} 1$

$\mathrm{C} 7-\mathrm{C} 12-\mathrm{C} 11-\mathrm{C} 10$

$\mathrm{C} 12-\mathrm{C} 7-\mathrm{C} 8-\mathrm{C} 9$

$\mathrm{C} 12-\mathrm{C} 7-\mathrm{Sn} 1-\mathrm{C} 1$

C12-C7-Sn1-C13

C12-C7-Sn1-Cl1

C12-C7-Sn1-O1

$\mathrm{C} 12-\mathrm{C} 11-\mathrm{C} 10-\mathrm{C} 9$

$\mathrm{C} 11-\mathrm{C} 10-\mathrm{C} 9-\mathrm{C} 8$

$\mathrm{C} 10-\mathrm{C} 9-\mathrm{C} 8-\mathrm{C} 7$

$\mathrm{C} 8-\mathrm{C} 7-\mathrm{C} 12-\mathrm{C} 11$

$\mathrm{C} 8-\mathrm{C} 7-\mathrm{Sn} 1-\mathrm{C} 1$

$\mathrm{C} 8-\mathrm{C} 7-\mathrm{Sn} 1-\mathrm{C} 13$

$\mathrm{C} 8-\mathrm{C} 7-\mathrm{Sn} 1-\mathrm{Cl1}$

$\mathrm{C} 8-\mathrm{C} 7-\mathrm{Sn} 1-\mathrm{O} 1$

$\mathrm{C} 13-\mathrm{C} 18-\mathrm{C} 17-\mathrm{C} 16$

$\mathrm{C} 18-\mathrm{C} 13-\mathrm{C} 14-\mathrm{C} 15$

$\mathrm{C} 18-\mathrm{C} 13-\mathrm{Sn} 1-\mathrm{C} 1$

C18-C13-Sn1-C7

$\mathrm{C} 18-\mathrm{C} 13-\mathrm{Sn} 1-\mathrm{C} 11$

C18-C13-Sn1-O1

$\mathrm{C} 18-\mathrm{C} 17-\mathrm{C} 16-\mathrm{C} 15$

$\mathrm{C} 17-\mathrm{C} 16-\mathrm{C} 15-\mathrm{C} 14$

$\mathrm{C} 16-\mathrm{C} 15-\mathrm{C} 14-\mathrm{C} 13$

$\mathrm{C} 14-\mathrm{C} 13-\mathrm{C} 18-\mathrm{C} 17$

C14-C13-Sn1-C1

C14-C13-Sn1-C7

C14-C13-Sn1-Cl1

$\mathrm{C} 14-\mathrm{C} 13-\mathrm{Sn} 1-\mathrm{O} 1$
120.0

120.0

120.0

120.0

120.0

0.0

0.0

$-61.0(4)$

$98.6(4)$

$-161.6(3)$

$22.6(4)$

0.0

0.0

0.0

0.0

$117.3(3)$

$-83.2(4)$

$16.6(3)$

$-159.1(4)$

0.0

0.0

46.3 (4)

$-112.8(4)$

$148.7(3)$

$-35.6(4)$

0.0

0.0

0.0

0.0

-134.1 (4)

$66.9(4)$

$-31.6(4)$

144.1 (4)

0.0

0.0

$-7.3(4)$

$151.8(4)$

-109.3 (4)

70.9 (4)

0.0

0.0

0.0

0.0

176.1 (3)

-24.8 (4)

74.1 (4)

-105.7 (4)
C13-Sn1-C7

C13-Sn1-Cl1

C13-Sn1-O1

Cl1-Sn1-O1

$120.2(2)$

94.63 (17)

80.4 (2)

175.07 (14)

$\begin{array}{ll}\mathrm{C} 23 \mathrm{~A}-\mathrm{C} 24 \mathrm{~A}-\mathrm{C} 25 \mathrm{~A}-\mathrm{C} 26 \mathrm{~A} & 0.0 \\ \mathrm{C} 24 \mathrm{~A}-\mathrm{C} 25 \mathrm{~A}-\mathrm{C} 26 \mathrm{~A}-\mathrm{C} 27 \mathrm{~A} & 0.0 \\ \mathrm{C} 25 \mathrm{~A}-\mathrm{C} 26 \mathrm{~A}-\mathrm{C} 27 \mathrm{~A}-\mathrm{C} 22 \mathrm{~A} & 0.0 \\ \mathrm{C} 27 \mathrm{~A}-\mathrm{C} 22 \mathrm{~A}-\mathrm{C} 23 \mathrm{~A}-\mathrm{C} 24 \mathrm{~A} & 0.0\end{array}$

$\mathrm{C} 27 \mathrm{~A}-\mathrm{C} 22 \mathrm{~A}-\mathrm{C} 21 \mathrm{~A}-\mathrm{N} 1 \quad 53(5)$

$\mathrm{C} 27 \mathrm{~A}-\mathrm{C} 22 \mathrm{~A}-\mathrm{C} 21 \mathrm{~A}-\mathrm{S} 1 \mathrm{~A}-68(5)$

$\mathrm{C} 20 \mathrm{~A}-\mathrm{C} 19-\mathrm{C} 20 \mathrm{~B}-\mathrm{S} 1 \mathrm{~B} \quad 68$ (6)

$\mathrm{C} 20 \mathrm{~A}-\mathrm{C} 19-\mathrm{N} 1-\mathrm{C} 21 \mathrm{~B} \quad-18$ (2)

$\mathrm{C} 20 \mathrm{~A}-\mathrm{C} 19-\mathrm{N} 1-\mathrm{C} 21 \mathrm{~A}-3(3)$

$\mathrm{C} 20 \mathrm{~A}-\mathrm{C} 19-\mathrm{N} 1-\mathrm{C} 28 \quad 165$ (2)

$\mathrm{C} 20 \mathrm{~A}-\mathrm{C} 19-\mathrm{O} 1-\mathrm{Sn} 1 \quad 39$ (3)

$\mathrm{C} 21 \mathrm{~A}-\mathrm{C} 22 \mathrm{~A}-\mathrm{C} 23 \mathrm{~A}-\mathrm{C} 24 \mathrm{~A}-173$ (4)

$\mathrm{C} 21 \mathrm{~A}-\mathrm{C} 22 \mathrm{~A}-\mathrm{C} 27 \mathrm{~A}-\mathrm{C} 26 \mathrm{~A} \quad 173$ (4)

$\mathrm{C} 22 \mathrm{~B}-\mathrm{C} 21 \mathrm{~B}-\mathrm{N} 1-\mathrm{C} 19 \quad-105$ (2)

$\mathrm{C} 22 \mathrm{~B}-\mathrm{C} 21 \mathrm{~B}-\mathrm{N} 1-\mathrm{C} 21 \mathrm{~A} \quad 169(17)$

$\mathrm{C} 22 \mathrm{~B}-\mathrm{C} 21 \mathrm{~B}-\mathrm{N} 1-\mathrm{C} 28 \quad 72$ (3)

$\mathrm{C} 22 \mathrm{~B}-\mathrm{C} 21 \mathrm{~B}-\mathrm{S} 1 \mathrm{~B}-\mathrm{C} 20 \mathrm{~B} \quad 100.0(17)$

$\mathrm{C} 22 \mathrm{~B}-\mathrm{C} 23 \mathrm{~B}-\mathrm{C} 24 \mathrm{~B}-\mathrm{C} 25 \mathrm{~B} \quad 0.0$

$\mathrm{C} 23 \mathrm{~B}-\mathrm{C} 22 \mathrm{~B}-\mathrm{C} 27 \mathrm{~B}-\mathrm{C} 26 \mathrm{~B} \quad 0.0$

$\mathrm{C} 23 \mathrm{~B}-\mathrm{C} 24 \mathrm{~B}-\mathrm{C} 25 \mathrm{~B}-\mathrm{C} 26 \mathrm{~B} \quad 0.0$

$\mathrm{C} 24 \mathrm{~B}-\mathrm{C} 25 \mathrm{~B}-\mathrm{C} 26 \mathrm{~B}-\mathrm{C} 27 \mathrm{~B} \quad 0.0$

$\mathrm{C} 25 \mathrm{~B}-\mathrm{C} 26 \mathrm{~B}-\mathrm{C} 27 \mathrm{~B}-\mathrm{C} 22 \mathrm{~B} \quad 0.0$

$\mathrm{C} 27 \mathrm{~B}-\mathrm{C} 22 \mathrm{~B}-\mathrm{C} 23 \mathrm{~B}-\mathrm{C} 24 \mathrm{~B} \quad 0.0$

$\mathrm{C} 28-\mathrm{C} 29-\mathrm{C} 30-\mathrm{C} 31 \quad-56.4(14)$

$\mathrm{C} 29-\mathrm{C} 28-\mathrm{C} 33-\mathrm{C} 32 \quad-58.0(12)$

$\mathrm{C} 29-\mathrm{C} 28-\mathrm{N} 1-\mathrm{C} 19 \quad 90.0(10)$

$\mathrm{C} 29-\mathrm{C} 28-\mathrm{N} 1-\mathrm{C} 21 \mathrm{~B} \quad-87.1(16)$

$\mathrm{C} 29-\mathrm{C} 28-\mathrm{N} 1-\mathrm{C} 21 \mathrm{~A} \quad-103(3)$

$\mathrm{C} 29-\mathrm{C} 30-\mathrm{C} 31-\mathrm{C} 32 \quad 57.4(16)$

$\mathrm{C} 30-\mathrm{C} 31-\mathrm{C} 32-\mathrm{C} 33 \quad-57.3(16)$

$\mathrm{C} 31-\mathrm{C} 32-\mathrm{C} 33-\mathrm{C} 28 \quad 57.0(14)$

$\mathrm{C} 33-\mathrm{C} 28-\mathrm{C} 29-\mathrm{C} 30 \quad 57.6(12)$

$\mathrm{C} 33-\mathrm{C} 28-\mathrm{N} 1-\mathrm{C} 19 \quad-143.8(8)$

$\mathrm{C} 33-\mathrm{C} 28-\mathrm{N} 1-\mathrm{C} 21 \mathrm{~B} \quad 39.1(17)$

$\mathrm{C} 33-\mathrm{C} 28-\mathrm{N} 1-\mathrm{C} 21 \mathrm{~A} \quad 23$ (3)

N1-C19-C20B-S1B $\quad-22.9(17)$

$\mathrm{N} 1-\mathrm{C} 19-\mathrm{C} 20 \mathrm{~A}-\mathrm{S} 1 \mathrm{~A} \quad-7(3)$

$\mathrm{N} 1-\mathrm{C} 19-\mathrm{O} 1-\mathrm{Sn} 1 \quad-157.1(6)$

$\mathrm{N} 1-\mathrm{C} 21 \mathrm{~B}-\mathrm{C} 22 \mathrm{~B}-\mathrm{C} 23 \mathrm{~B} \quad-148.8(19)$

$\mathrm{N} 1-\mathrm{C} 21 \mathrm{~B}-\mathrm{C} 22 \mathrm{~B}-\mathrm{C} 27 \mathrm{~B} \quad 41$ (3)

N1-C21B-S1B-C20B $\quad-26.7$ (18)

$\mathrm{N} 1-\mathrm{C} 21 \mathrm{~A}-\mathrm{S} 1 \mathrm{~A}-\mathrm{C} 20 \mathrm{~A}-13$ (4) 


$\begin{array}{ll}\mathrm{C} 19-\mathrm{C} 20 \mathrm{~B}-\mathrm{S} 1 \mathrm{~B}-\mathrm{C} 21 \mathrm{~B} & 27.5(17) \\ \mathrm{C} 19-\mathrm{C} 20 \mathrm{~A}-\mathrm{S} 1 \mathrm{~A}-\mathrm{C} 21 \mathrm{~A} & 12(4) \\ \mathrm{C} 19-\mathrm{O} 1-\mathrm{S} 1-\mathrm{C} 1 & 175.4(7) \\ \mathrm{C} 19-\mathrm{O} 1-\mathrm{Sn} 1-\mathrm{C} 7 & -65.6(7) \\ \mathrm{C} 19-\mathrm{O} 1-\mathrm{S} 1-\mathrm{C} 13 & 55.7(7) \\ \mathrm{C} 19-\mathrm{O} 1-\mathrm{S} 1-\mathrm{C} 11 & 53.7(18) \\ \mathrm{C} 20 \mathrm{~B}-\mathrm{C} 19-\mathrm{C} 20 \mathrm{~A}-\mathrm{S} 1 \mathrm{~A} & -104(8) \\ \mathrm{C} 20 \mathrm{~B}-\mathrm{C} 19-\mathrm{N} 1-\mathrm{C} 21 \mathrm{~B} & 2.6(17) \\ \mathrm{C} 20 \mathrm{~B}-\mathrm{C} 19-\mathrm{N} 1-\mathrm{C} 21 \mathrm{~A} & 18(3) \\ \mathrm{C} 20 \mathrm{~B}-\mathrm{C} 19-\mathrm{N} 1-\mathrm{C} 28 & -174.6(13) \\ \mathrm{C} 20 \mathrm{~B}-\mathrm{C} 19-\mathrm{O} 1-\mathrm{S} 1 & 17.1(17) \\ \mathrm{C} 21 \mathrm{~B}-\mathrm{C} 22 \mathrm{~B}-\mathrm{C} 23 \mathrm{~B}-\mathrm{C} 24 \mathrm{~B} & -170(2) \\ \mathrm{C} 21 \mathrm{~B}-\mathrm{C} 22 \mathrm{~B}-\mathrm{C} 27 \mathrm{~B}-\mathrm{C} 26 \mathrm{~B} & 170(2) \\ \mathrm{C} 22 \mathrm{~A}-\mathrm{C} 23 \mathrm{~A}-\mathrm{C} 24 \mathrm{~A}-\mathrm{C} 25 \mathrm{~A} & 0.0 \\ \mathrm{C} 22 \mathrm{~A}-\mathrm{C} 21 \mathrm{~A}-\mathrm{N} 1-\mathrm{C} 19 & -116(3) \\ \mathrm{C} 22 \mathrm{~A}-\mathrm{C} 21 \mathrm{~A}-\mathrm{N} 1-\mathrm{C} 21 \mathrm{~B} & -15(12) \\ \mathrm{C} 22 \mathrm{~A}-\mathrm{C} 21 \mathrm{~A}-\mathrm{N} 1-\mathrm{C} 28 & 77(4) \\ \mathrm{C} 22 \mathrm{~A}-\mathrm{C} 21 \mathrm{~A}-\mathrm{S} 1 \mathrm{~A}-\mathrm{C} 20 \mathrm{~A} & 109(4) \\ \mathrm{C} 23 \mathrm{~A}-\mathrm{C} 22 \mathrm{~A}-\mathrm{C} 27 \mathrm{~A}-\mathrm{C} 26 \mathrm{~A} & 0.0 \\ \mathrm{C} 23 \mathrm{~A}-\mathrm{C} 22 \mathrm{~A}-\mathrm{C} 21 \mathrm{~A}-\mathrm{N} 1 & -134(3) \\ \mathrm{C} 23 \mathrm{~A}-\mathrm{C} 22 \mathrm{~A}-\mathrm{C} 21 \mathrm{~A}-\mathrm{S} 1 \mathrm{~A} & 106(4) \\ \end{array}$

$\begin{array}{ll}\mathrm{N} 1-\mathrm{C} 28-\mathrm{C} 29-\mathrm{C} 30 & -175.3(8) \\ \mathrm{N} 1-\mathrm{C} 28-\mathrm{C} 33-\mathrm{C} 32 & 176.3(9) \\ \mathrm{O} 1-\mathrm{C} 19-\mathrm{C} 20 \mathrm{~B}-\mathrm{S} 1 \mathrm{~B} & 162.3(11) \\ \mathrm{O} 1-\mathrm{C} 19-\mathrm{C} 20 \mathrm{~A}-\mathrm{S} 1 \mathrm{~A} & 158.2(17) \\ \mathrm{O} 1-\mathrm{C} 19-\mathrm{N} 1-\mathrm{C} 21 \mathrm{~B} & 177.3(15) \\ \mathrm{O} 1-\mathrm{C} 19-\mathrm{N} 1-\mathrm{C} 21 \mathrm{~A} & -168(3) \\ \mathrm{O} 1-\mathrm{C} 19-\mathrm{N} 1-\mathrm{C} 28 & 0.1(12) \\ \mathrm{S} 1 \mathrm{~A}-\mathrm{C} 21 \mathrm{~A}-\mathrm{N} 1-\mathrm{C} 19 & 11(4) \\ \mathrm{S} 1 \mathrm{~A}-\mathrm{C} 21 \mathrm{~A}-\mathrm{N} 1-\mathrm{C} 21 \mathrm{~B} & 112(17) \\ \mathrm{S} 1 \mathrm{~A}-\mathrm{C} 21 \mathrm{~A}-\mathrm{N} 1-\mathrm{C} 28 & -156(2) \\ \mathrm{S} 1 \mathrm{~B}-\mathrm{C} 21 \mathrm{~B}-\mathrm{C} 22 \mathrm{~B}-\mathrm{C} 23 \mathrm{~B} & 92.4(18) \\ \text { S1B-C21B-C22B-C27B } & -78(2) \\ \text { S1B-C21B-N1-C19 } & 17.8(19) \\ \text { S1B-C21B-N1-C21A } & -68(14) \\ \text { S1B-C21B-N1-C28 } & -165.0(11) \\ \text { Sn1-C1-C2-C3 } & 178.2(4) \\ \text { Sn1-C1-C6-C5 } & -178.3(4) \\ \text { Sn1-C7-C12-C11 } & 179.7(4) \\ \text { Sn1-C7-C8-C9 } & -179.7(4) \\ \text { Sn1-C13-C18-C17 } & -176.6(4) \\ \text { Sn1-C13-C14-C15 } & 176.5(4) \\ \end{array}$

